\title{
A multicenter, randomized, rater-blinded, parallel-group, phase 3 study to compare the efficacy, safety, and immunogenicity of biosimilar RGB-10 and reference once-daily teriparatide in patients with osteoporosis
}

\author{
H. Hagino ${ }^{1} \cdot$ R. Narita ${ }^{2} \cdot$ Y. Yokoyama ${ }^{2} \cdot$ M. Watanabe ${ }^{2} \cdot$ M. Tomomitsu ${ }^{2}$ \\ Received: 15 January 2019 / Accepted: 28 May 2019 / Published online: 26 June 2019 \\ (C) The Author(s) 2019
}

\begin{abstract}
Summary The efficacy and safety of RGB-10 and reference teriparatide were evaluated in a randomized 52-week study in 250 patients with osteoporosis at high risk of fracture. RGB-10 was equivalent to reference teriparatide in efficacy and had a comparable safety profile.

Introduction RGB-10 is the first biosimilar teriparatide authorized in the European Union. This multicenter, randomized, raterblinded, parallel-group phase 3 study evaluated equivalence in efficacy and compared safety between RGB-10 and reference teriparatide in patients with osteoporosis at high risk of fracture for registration in Japan.

Methods Ambulatory postmenopausal women and men ( $\geq 55$ years of age) with osteoporosis at high risk of fracture were randomized 1:1 to receive either RGB-10 or reference teriparatide $20 \mu \mathrm{g}$ once daily via subcutaneous self-injection for 52 weeks. The primary efficacy endpoint was the percent change from baseline to 52 weeks in lumbar spine (L2-L4) bone mineral density (BMD). Safety outcomes and immunogenicity were also assessed.

Results In total, 250 patients (125 in each group) were randomized. The percent change from baseline to 52 weeks in lumbar spine (L2-L4) BMD (mean \pm standard deviation) was $8.94 \% \pm 6.19 \%$ in the RGB-10 group and $9.65 \% \pm 6.22 \%$ in the reference teriparatide group. The estimated between-group difference ( $95 \%$ confidence interval) was $-0.65 \%(-2.17 \%$ to $-0.87 \%)$ within the pre-specified equivalence margin $( \pm 2.8 \%)$, which indicates equivalence in efficacy between the two groups. Changes in BMD at lumbar spine (L1-L4), femoral neck, and total hip and serum procollagen type I amino-terminal propeptide were also similar between the groups. Safety profiles, including immunogenicity, were comparable.

Conclusions The therapeutic equivalence of RGB-10 to reference teriparatide was demonstrated. RGB-10 had comparable safety profile to that of reference teriparatide.
\end{abstract}

Keywords Biosimilar $\cdot$ BMD $\cdot$ Clinical trial $\cdot$ Osteoporosis $\cdot$ RGB-10 $\cdot$ Teriparatide

Electronic supplementary material The online version of this article (https://doi.org/10.1007/s00198-019-05038-y) contains supplementary material, which is available to authorized users.

H. Hagino

hagino@tottori-u.ac.jp

1 School of Health Science, Faculty of Medicine, Tottori University, 86 Nishicho, Yonago City, Tottori 683-8503, Japan

2 Mochida Pharmaceutical Co. Ltd, Tokyo, Japan

\section{Introduction}

Recombinant human teriparatide (rhPTH [1-34]), produced in E. coli, is the 1-34 N-terminal active fragment of endogenous 84-amino acid human parathyroid hormone (PTH). Teriparatide exerts the same physiological actions on bone and kidney as PTH, including the elevation of serum calcium levels through indirect stimulation of bone resorption by enhancing the production of receptor activator of NF-KB ligand (RANKL) and inhibiting the expression of osteoprotegerin, 
promoting the re-absorption of calcium and excretion of phosphate from the renal tubule, and promoting 1,25dihydroxyvitamin D production, which indirectly increases calcium absorption from the intestine [1-4].

Effects of teriparatide on the skeleton differ depending on the pattern of systemic exposure: intermittent administration of teriparatide promotes bone formation, increases bone mineral density (BMD), and restores bone microarchitecture, resulting in fracture prevention, whereas continuous exposure to teriparatide promotes bone resorption over bone formation and results in a reduction in bone mass [3,5]. Taking advantage of the bone formation effect resulting from intermittent administration, $20 \mu \mathrm{g}$ once-daily injection of teriparatide has been approved for the treatment of osteoporosis in the USA, EU, and Japan, among other countries. Although multiple studies have demonstrated that teriparatide can increase BMD and prevent vertebral and non-vertebral fragility fractures [6-9], its high cost as a biological medicinal product places a heavy burden on individual patients and on the healthcare systems.

A biosimilar is a biological medicine with high similarity to another biological medicine already approved by a Stringent Regulatory Authority (a so-called 'reference medicine') [10, 11]. Regulatory guidelines in the EU, USA, and Japan require similarity in terms of quality characteristics, biological activity, safety, and efficacy to be demonstrated between the biosimilar and the reference medicine in a comprehensive comparability exercise [12-14]. Given their relatively low price compared with reference medicinal products, biosimilars represent a more affordable but equally efficacious and safe treatment option for patients and healthcare systems, thus increasing patients' access to treatment.

RGB-10, manufactured by Gedeon Richter Plc., has been developed as a biosimilar to reference teriparatide, and its active ingredient has an amino acid sequence identical to that of teriparatide. RGB-10 also shows a high level of similarity to teriparatide with respect to quality characteristics, pharmacological effects, and toxicities (data on file). Furthermore, a comparative pharmacokinetic (PK) study sponsored by Gedeon Richter Plc. and conducted in healthy female volunteers in the United Kingdom (NCT02223416) demonstrated equivalence in terms of PK characteristics following administration of a single dose of RGB-10 or the reference teriparatide [15]. Based on these results, RGB-10 was approved by the European Medicines Agency (EMA) in 2017 [16] for the same indications as those stated in the EU label for reference teriparatide.

The objectives of the current phase 3 study were to evaluate equivalence in efficacy and compare safety between RGB-10 and reference teriparatide in patients with osteoporosis at high risk of fracture.

\section{Methods}

\section{Study design and treatment}

A multicenter, randomized, active comparator-controlled, rater-blinded, parallel-group phase 3 study (JapicCTI-163208) was conducted at 34 sites in Japan. After a screening period of 2 weeks, patients were randomly assigned 1:1 to receive either RGB-10 (manufactured by Gedeon Richter Plc, Debrecen, Hungary) or reference teriparatide (Forteo®; Eli Lilly Japan K.K., Kobe, Japan) $20 \mu \mathrm{g}$ once daily via subcutaneous self-injection for a treatment period of 52 weeks, followed by a follow-up period of 2 weeks. A multi-dose pen injector (ServoPen Fix ${ }^{\circledR}$; Ypsomed AG, Burgdorf, Switzerland) was used for injection of RGB-10. This device has the same injection procedure as that of reference teriparatide and was confirmed to have a similar dose accuracy. All patients received daily oral supplementation of $610 \mathrm{mg}$ calcium and $400 \mathrm{IU}$ vitamin $\mathrm{D}$ as basal treatment throughout the screening and treatment period. Concomitant use of medication that can affect bone metabolism or cause secondary osteoporosis, such as bisphosphonates and systemic corticosteroids, was prohibited.

\section{Patients}

Postmenopausal women and men (aged $\geq 55$ years) who were ambulatory with sufficient physical and cognitive ability for self-injection were eligible for this study if they met any of the following criteria:

BMD at lumbar spine (L2-L4) $<80 \%$ of young adult mean (YAM; mean BMD in healthy Japanese women aged 2044 years) [17] (T-score $<-1.7$ ) with at least 1 vertebral fragility fracture; BMD at lumbar spine (L2-L4) $<70 \%$ of YAM (Tscore $<-2.6)$ and aged $\geq 65$ years; or BMD at lumbar spine (L2-L4) $<65 \%$ of YAM (T-score <-3.0). Exclusion criteria included suspected secondary osteoporosis; metabolic bone disease or disease causing a decrease in bone volume other than osteoporosis; previous or concurrent renal/urinary calculus or malignant tumor; previous radiation therapy to the skeleton; high alkaline phosphatase of unknown cause; abnormalities in laboratory test parameters including estimated glomerular filtration rate, corrected serum calcium, or intact PTH; or any condition that could affect the assessment of lumbar spine or femoral BMD by dual-energy $\mathrm{x}$-ray absorptiometry (DXA). Patients were also excluded from the study if they had received medication affecting bone metabolism including bisphosphonate within the 22-week period prior to screening, plus the duration equal to its dosing interval (e.g., 22 weeks + 7 days for weekly bisphosphonate); calcitonin, ipriflavone, sex hormone preparations, anabolic hormone preparations, vitamin $\mathrm{K}$, selective estrogen receptor modulators, or eldecalcitol within 6 weeks; alfacalcidol or calcitriol within 
2 weeks; or denosumab or PTH preparation at any time prior to the start of the screening period.

\section{Randomization and blinding}

After screening, patients who met all eligibility criteria were randomly assigned to either the RGB-10 or reference teriparatide group in a 1:1 ratio within randomization factors by a central registration system using a dynamic allocation method. The randomization factors were lumbar spine (L2L4) BMD at screening $(<65 \%,<70 \%$, or $<80 \%$ of YAM) and presence of prior treatment with bisphosphonates. The number of patients allocated to each treatment group was also balanced within each study site.

As the injection devices for RGB-10 and reference teriparatide differed in appearance, this study was performed in a rater-blinded manner. Raters such as investigators and radiographers for the measurement of $\mathrm{BMD}$ or the evaluation of fractures were therefore blinded. To maintain raterblinding, especially among investigators, all procedures associated with the handling of investigational products at study sites were conducted by unblinded site staff, and patients were instructed not to disclose any information to raters that could identify the treatment group to which they were allocated.

\section{Efficacy endpoints and measurement}

The primary efficacy endpoint was the percent change from baseline to 52 weeks in lumbar spine (L2-L4) BMD. Secondary efficacy endpoints included percent change from baseline to 52 weeks in BMD at lumbar spine (L1-L4), total hip, and femoral neck; percent change from baseline to 52 weeks in the serum bone formation marker procollagen type I amino-terminal propeptide (P1NP); and incidence of fractures.

BMD at lumbar spine, total hip, and femoral neck were measured at the start of the screening period and again at baseline, week 12 , week 24 , and week 52 /discontinuation by DXA using the Explorer, Discovery, or Horizon systems (Hologic Inc., Marlborough, MA, USA). BMD measurements were analyzed centrally (Bioclinica Inc., Princeton, NJ, USA) in a blinded manner. To maintain densitometry stability during the study, longitudinal quality control was performed using a quality control phantom at each study site. In addition, to ensure consistency across study sites, BMD data at each site were corrected as necessary based on the measurements of a standard spine phantom.

Serum P1NP was measured at the start of the screening period and again at baseline, week 4 , week 12 , week 24 , and week 52/discontinuation using an electrochemiluminescence immunoassay (Modular Analytics E; Roche Diagnostics K.K., Tokyo, Japan). All samples were centrally analyzed (LSI Medience Corp., Tokyo, Japan).
The incidence of vertebral fractures was assessed using a semi-quantitative method by both the central assessment facility (Bioclinica Inc., Princeton, NJ, USA) and the study investigators. Assessments were based on radiographs obtained at the start of the screening period and at week 24 and week 52/discontinuation. Investigators also assessed the incidence of non-vertebral fractures based on radiographs obtained when a fracture was suspected. In the assessment by investigators, the cause of vertebral and non-vertebral fractures (fragility fracture or traumatic fracture) was evaluated.

\section{Safety endpoints and measurements}

Safety endpoints were the incidence of adverse events (AEs) in the treatment period and follow-up period; abnormalities in laboratory test parameters, electrocardiograms (ECG), vital signs, and weight; and immunogenicity. AEs were coded using the Medical Dictionary for Regulatory Activities (MedDRA, version 20.1). Samples for laboratory testing, vital signs, and weight were collected at screening, baseline, week 4 , week 12, week 24, and week 52/discontinuation. ECG was performed at screening, week 24 , and week 52/discontinuation. For the evaluation of immunogenicity, anti-teriparatide antibodies and neutralizing activities were analyzed in serum samples collected at baseline and week 52/discontinuation. Anti-teriparatide antibodies were detected with a validated electrochemiluminescent immunoassay using the Meso Scale Discovery platform (Meso Scale Diagnostics, LLC., Rockville, MD, USA) and biotinylated RGB-10 and SULFO-TAG-labeled RGB-10. A validated cell-based assay (CatchPoint cyclic-AMP fluorescent assay kit; Molecular Devices Japan K.K., Tokyo, Japan) was used to measure neutralizing activities. Assessments for neutralizing activities were conducted only in samples positive for anti-teriparatide antibodies. Samples for laboratory testing and immunogenicity analysis were centrally analyzed at the same facility as for P1NP (LSI Medience Corp., Tokyo, Japan).

\section{Statistical analyses}

The equivalence margin of the primary efficacy endpoint, percent change from baseline to 52 weeks in lumbar spine (L2L4) BMD, was predetermined as $\pm 2.8 \%$, the most conservative range based on an expected effect size and minimum clinically significant change: the effect size, the mean difference between the primary efficacy endpoint in the active group and the placebo group, was estimated as $9.78 \%$ from the results of a previous phase 3 study of reference teriparatide in Japan [7]. The minimum clinically significant change, the least significant change in lumbar spine BMD by DXA, was estimated to be $2.8-5.6 \%$, given the coefficient of variation of DXA measurement for lumbar spine BMD of 1-2\% [18] and a confidence level of $95 \%$. 
A sample size of 236 randomized patients (118 patients per group) was calculated as follows to ensure sufficient power to demonstrate equivalence for the primary efficacy endpoint between the groups as well as to assure 100 subjects with safety data for 52 weeks: a sample size of 194 patients (97 patients per group) was estimated to demonstrate equivalence between the groups for the primary efficacy endpoint with two one-sided tests at an alpha level of $2.5 \%$ and $90 \%$ statistical power. It was assumed that the between-group difference was $0.0 \%$ and that the common standard deviation (SD) across the treatment groups was $5.36 \%$ based on the results of the previous phase 3 study of reference teriparatide in Japan [7]; a sample size of 236 patients (118 patients per group) was estimated to assure 100 subjects with safety data for 52 weeks, where the dropout rate was assumed to be $15 \%$ based on the previous phase 3 study [7].

The primary analysis set for efficacy analysis was the full analysis set (FAS), and the supplementary analysis set for efficacy analysis was the per protocol set (PPS). Safety endpoints were analyzed in the safety analysis set (SS). Detailed criteria for the FAS, PPS, and SS are provided in Online Resource 1.

Analysis of covariance (ANCOVA) was performed on the percent change in BMD and P1NP using corresponding baseline value and previous treatment with bisphosphonate as covariates. The point estimate of the difference in least squares mean (LS mean) between the groups as well as its two-sided 95\% CI was calculated.

The last observation carried forward method (LOCF) was used for imputation of missing data for the efficacy endpoint. No imputation was performed on the safety endpoint.

All analyses were performed using SAS version 9.2 (SAS Institute Inc., Cary, NC, USA).

\section{Results}

A total of 369 patients were screened, of which 250 were randomized to either RGB-10 or reference teriparatide treatment groups ( $n=125$ in each group). A total of 219 patients completed the 52-week treatment period. The most common reason for discontinuation in both groups was occurrence of AEs (RGB-10, $n=15$; reference teriparatide, $n=11$ ) (Fig. 1). The FAS included all 250 patients, and 32 of these patients were excluded from the PPS. No patients were excluded from the SS. The baseline demographics and characteristics of patients were balanced between the groups (Table 1); $96.4 \%$ $(n=241)$ of the randomized patients were female and the mean patient age $( \pm \mathrm{SD})$ was $70.4 \pm 6.4$ years. Lumbar spine (L2-L4) BMD at baseline (mean \pm SD) was $0.6275 \pm$ $0.0752 \mathrm{~g} / \mathrm{cm}^{2}$. The percentage of patients with prior treatment with bisphosphonates was $4.4 \%(n=11)$. Oral minodronate, risedronate, alendronate, and intravenous ibandronate were reported as prior treatment with bisphosphonates. All randomized patients were of Asian ethnicity.

\section{Efficacy}

\section{BMD}

The percent change from baseline to 52 weeks in lumbar spine (L2-L4) BMD (mean \pm SD), the primary efficacy endpoint, was $8.94 \% \pm 6.19 \%$ in the RGB- 10 group and $9.65 \% \pm 6.22 \%$ in the reference teriparatide group (Table 2). The estimated between-group difference (LS mean) was $-0.65 \%$ (95\% CI: $-2.17 \%$ to $0.87 \%$ ). As the two-sided $95 \%$ CI of the betweengroup difference was within the pre-specified equivalence margin $( \pm 2.8 \%)$, equivalence in efficacy between the two groups was demonstrated. A similar result was found in the PPS ( $-0.31 \%$ [95\% CI: $-1.80 \%$ to $1.18 \%])$. The mean percent change from baseline in lumbar spine (L2-L4) BMD longitudinally increased for both RGB-10 and reference teriparatide through the treatment period, and percent change from baseline was similar at all time points between the two groups (Fig. 2a).

Changes in BMD at other skeletal sites were also similar between the groups (Fig. 2b-d). The percent change from baseline to 52 weeks in BMD at lumbar spine (L1-L4), femoral neck, and total hip (mean \pm SD) were as follows: $9.04 \% \pm$ $6.15 \%$ in the RGB-10 group and $9.66 \% \pm 6.13 \%$ in the reference teriparatide group for lumbar spine (L1-L4); $1.48 \% \pm$ $4.36 \%$ in the RGB-10 group and $1.40 \% \pm 4.54 \%$ in the reference teriparatide group for femoral neck; and $1.31 \% \pm 3.71 \%$ in the RGB-10 group and $1.51 \% \pm 3.96 \%$ in the reference teriparatide group for total hip (Table 2).

\section{Bone formation marker (P1NP)}

Overall, the change in serum P1NP was similar between the groups, and a rapid increase in P1NP level following treatment was observed in both groups (Fig. 2e). The percent change from baseline to 52 weeks in serum P1NP (mean \pm SD) was $269.75 \% \pm 258.35 \%$ in the RGB-10 group and $230.84 \% \pm 267.29 \%$ in the reference teriparatide group. The estimated between-group difference (LS mean) was 35.06\% (95\% CI: $-28.01 \%$ to $98.13 \%$ ) (Table 2).

\section{Fractures}

A summary of the incidence of vertebral and non-vertebral fractures evaluated by the central assessment facility and the investigator is presented in Online Resource 2. Overall, the incidence of vertebral and non-vertebral fractures was numerically similar between the groups. The incidence of new vertebral fractures by central assessment was $0.9 \%$ (1/117 patients) in the RGB-10 group and $0.8 \%(1 / 124)$ in the reference 
Fig. 1 Patient disposition. BMD, bone mineral density; FAS, full analysis set; PPS, per protocol set

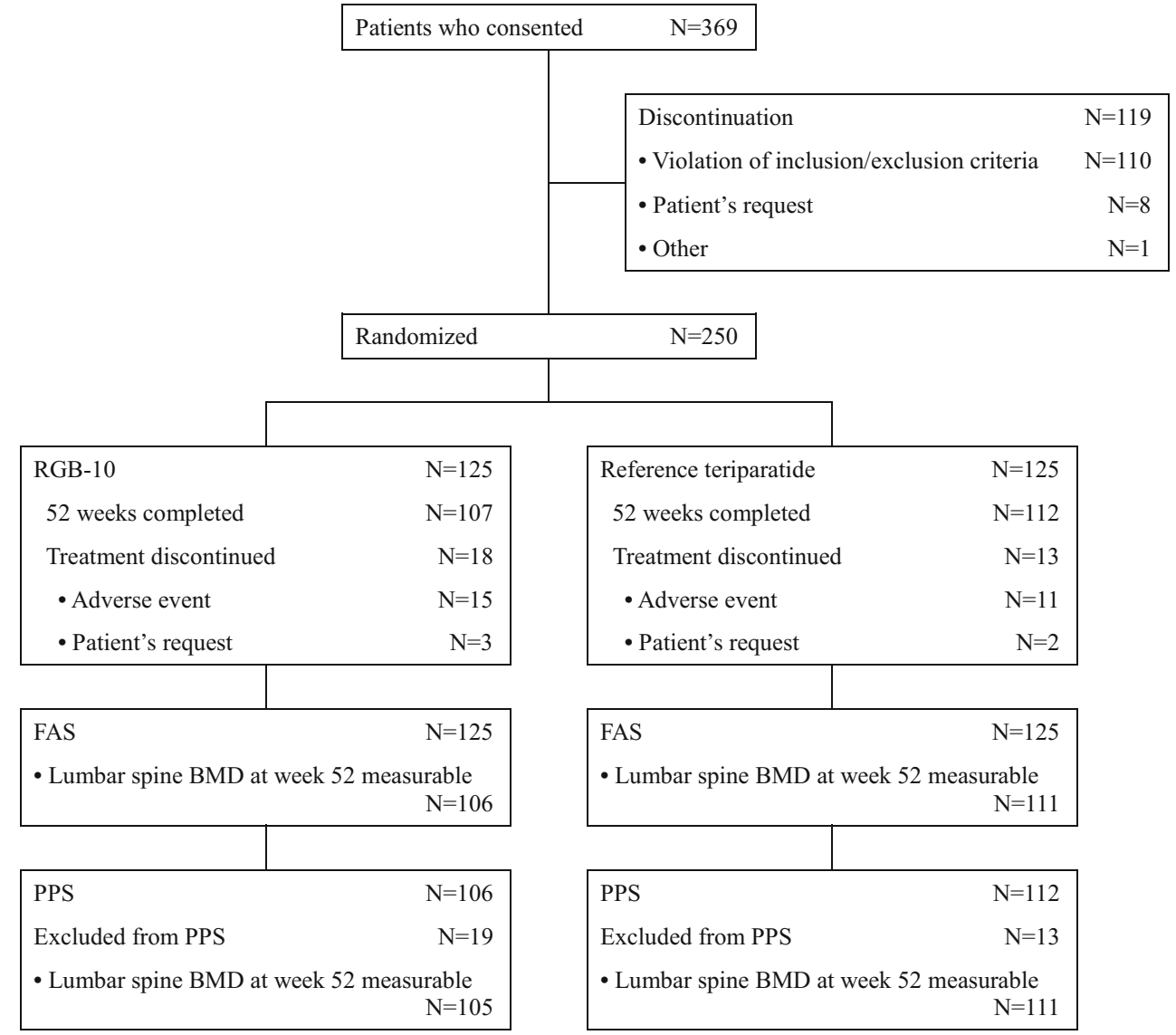

teriparatide group. No worsening of vertebral fractures was identified by central assessment.

The incidence of non-vertebral fractures was $2.4 \%(3 / 125)$ in the RGB-10 group and 1.6\% (2/125) in the reference teriparatide group; fragility fractures were reported for $0.9 \%$ $(1 / 125)$ of patients in the RGB-10 group and 1.6\% (2/125) in the reference teriparatide group.

\section{Safety}

There were no major differences between the treatment groups in the incidence of AEs, serious AEs (SAEs), or AEs leading to study discontinuation (Table 3). A total of $85.6 \%(n=107)$ of patients in each treatment group experienced one or more AEs, most of which were mild or moderate in severity. No AEs leading to death were reported. SAEs were reported in $2.4 \%(n=3)$ and $4.8 \%(n=6)$ of patients in the RGB-10 group and the reference teriparatide group, respectively. No SAEs were considered related to the investigational drug. AEs leading to study discontinuation were reported in $12.0 \%(n=15)$ and $8.8 \%(n=11)$ of patients receiving RGB-10 and reference teriparatide, respectively (Online Resource 3 ).

The most frequently reported AEs were nasopharyngitis (25.6\%, $n=32)$, injection site erythema $(10.4 \%, n=13)$, and nausea $(10.4 \%, n=13)$ in the RGB-10 group, and nasopharyngitis $(28.8 \%, n=36)$, blood uric acid increased $(9.6 \%, n=12)$, and headache $(8.8 \%, n=11)$ in the reference teriparatide group. The incidences of frequently reported AEs ( $>2 \%$; Online Resource 4) were comparable between the treatment groups. The incidence of injection site bruising was numerically higher in the RGB-10 group $(9.6 \%, n=12)$ compared with the reference teriparatide group $(3.2 \%, n=4)$.

The incidence of hypercalcaemia was $0.8 \%(n=1)$ in the RGB-10 group and $0.0 \%$ in the reference teriparatide group. The highest value of serum calcium concentration in a patient who developed hypercalcaemia was $11.3 \mathrm{mg} / \mathrm{dL}$, which was above the upper limit of the normal range $(10.4 \mathrm{mg} / \mathrm{dL})$. Following temporary discontinuation of basal treatment, this patient had recovered from the event by approximately 2.5 months after onset. No cases of osteosarcoma or nonuremic calciphylaxis, which can potentially be induced by teriparatide treatment, were reported.

Regarding laboratory test parameters, ECG, vital signs, and body weight, there were no apparent differences between the two groups in terms of the degree of change or the incidence and severity of abnormalities. Furthermore, no clinically significant changes in these parameters were observed.

The incidence of newly developed anti-teriparatide antibodies after the administration of investigational drug was $0.0 \%$ in the RGB-10 group and $0.8 \%(n=1)$ in the 
Table 1 Baseline patient demographics and characteristics (full analysis set)

\begin{tabular}{|c|c|c|c|}
\hline Variable & RGB-10 $(n=125)$ & Reference teriparatide $\left(n=125^{\mathrm{a}}\right)$ & Overall $\left(n=250^{\mathrm{b}}\right)$ \\
\hline Age, years & $70.5 \pm 6.0$ & $70.3 \pm 6.8$ & $70.4 \pm 6.4$ \\
\hline Female, $n(\%)$ & $121(96.8)$ & $120(96.0)$ & $241(96.4)$ \\
\hline Height, cm & $151.13 \pm 6.75$ & $151.74 \pm 6.33$ & $151.43 \pm 6.53$ \\
\hline Weight, kg & $48.42 \pm 7.56$ & $49.16 \pm 7.90$ & $48.79 \pm 7.72$ \\
\hline BMI, kg/m² & $21.23 \pm 3.24$ & $21.35 \pm 3.15$ & $21.29 \pm 3.19$ \\
\hline Years after menopause, years & $20.0 \pm 7.3$ & $19.1 \pm 8.4$ & $19.5 \pm 7.8$ \\
\hline Prior osteoporosis treatment, $n(\%)$ & $31(24.8)$ & $34(27.2)$ & $65(26.0)$ \\
\hline Prior osteoporosis treatment with bisphosphonates, $n(\%)$ & $6(4.8)$ & $5(4.0)$ & $11(4.4)$ \\
\hline \multicolumn{4}{|l|}{ Prevalent vertebral fractures ${ }^{c}$} \\
\hline $0, n(\%)$ & 77 (61.6) & $88(70.4)$ & $165(66.0)$ \\
\hline $1, n(\%)$ & $37(29.6)$ & $29(23.2)$ & $66(26.4)$ \\
\hline$\geq 2, n(\%)$ & $11(8.8)$ & $8(6.4)$ & $19(7.6)$ \\
\hline Lumbar spine (L2-L4) BMD, g/ $\mathrm{cm}^{2}$ & $0.6276 \pm 0.0758$ & $0.6273 \pm 0.0748$ & $0.6275 \pm 0.0752$ \\
\hline \multicolumn{4}{|l|}{ Percent YAM in lumbar spine (L2-L4) BMD } \\
\hline$<65 \%, n(\%)$ & $74(59.2)$ & $82(65.6)$ & $156(62.4)$ \\
\hline$\geq 65-<70 \%, n(\%)$ & $39(31.2)$ & $30(24.0)$ & $69(27.6)$ \\
\hline$\geq 70 \%, n(\%)$ & $12(9.6)$ & $13(10.4)$ & $25(10.0)$ \\
\hline Lumbar spine (L1-L4) BMD, g/ $\mathrm{cm}^{2}$ & $0.6175 \pm 0.0722$ & $0.6172 \pm 0.0693$ & $0.6174 \pm 0.0706$ \\
\hline Femoral neck BMD, $\mathrm{g} / \mathrm{cm}^{2}$ & $0.5035 \pm 0.0754$ & $0.5041 \pm 0.0746$ & $0.5038 \pm 0.0748$ \\
\hline Total hip BMD, $\mathrm{g} / \mathrm{cm}^{2}$ & $0.6177 \pm 0.0876$ & $0.6157 \pm 0.0932$ & $0.6167 \pm 0.0903$ \\
\hline Serum P1NP, $\mu \mathrm{g} / \mathrm{L}$ & $54.90 \pm 20.10$ & $55.93 \pm 19.22$ & $55.41 \pm 19.63$ \\
\hline 25-OH-vitamin D2, ng/mL & $4.00 \pm 0.00$ & $4.03 \pm 0.29$ & $4.02 \pm 0.20$ \\
\hline 25-OH-vitamin D3, ng/mL & $20.61 \pm 7.18$ & $19.99 \pm 6.02$ & $20.30 \pm 6.62$ \\
\hline
\end{tabular}

Data for continuous variables are expressed as mean $\pm \mathrm{SD}$

${ }^{\mathrm{a}} n=124$ for serum P1NP

${ }^{\mathrm{b}} n=249$ for serum P1NP

${ }^{\mathrm{c}}$ Assessed by the central assessment facility

$B M D$ bone mineral density, $B M I$ body mass index, $P I N P$ procollagen type I amino-terminal propeptide, YAM young adult mean

Table 2 Percent change and between-group difference from baseline to 52 weeks in BMD and serum P1NP

\begin{tabular}{|c|c|c|c|}
\hline & \multicolumn{2}{|c|}{ Percent change from baseline to 52 weeks (mean $\pm \mathrm{SD}$ ) } & \multirow{2}{*}{$\begin{array}{l}\text { Estimated between-group difference (LS mean } \\
[95 \% \mathrm{CI}])^{\mathrm{a}}\end{array}$} \\
\hline & $\begin{array}{l}\text { RGB-10 (BMD, } n=121 ; \mathrm{P} 1 \mathrm{NP}, \\
n=123)\end{array}$ & $\begin{array}{l}\text { Reference teriparatide (BMD, } n=124 \text {; } \\
\text { P1NP, } n=123 \text { ) }\end{array}$ & \\
\hline \multicolumn{4}{|l|}{ BMD } \\
\hline $\begin{array}{l}\text { Lumbar spine } \\
\text { (L2-L4) }\end{array}$ & $8.94 \pm 6.19$ & $9.65 \pm 6.22$ & $-0.65(-2.17$ to 0.87$)$ \\
\hline $\begin{array}{l}\text { Lumbar spine } \\
\text { (L1-L4) }\end{array}$ & $9.04 \pm 6.15$ & $9.66 \pm 6.13$ & $-0.56(-2.05$ to 0.93$)$ \\
\hline Femoral neck & $1.48 \pm 4.36$ & $1.40 \pm 4.54$ & $0.10(-0.98$ to 1.19$)$ \\
\hline Total hip & $1.31 \pm 3.71$ & $1.51 \pm 3.96$ & $-0.12(-1.01$ to 0.77$)$ \\
\hline Serum P1NP & $269.75 \pm 258.35$ & $230.84 \pm 267.29$ & $35.06(-28.01$ to 98.13$)$ \\
\hline
\end{tabular}

${ }^{a}$ Adjusted by baseline value and prior treatment with bisphosphonates

$B M D$ bone mineral density, $C I$ confidence interval, $L S$ mean least squares mean, $P 1 N P$ procollagen type I amino-terminal propeptide 
Fig. 2 Percent change from baseline to 52 weeks in BMD at a lumbar spine (L2-L4), b lumbar spine (L1-L4), c femoral neck, and $\mathbf{d}$ total hip, and in $\mathbf{e}$ serum P1NP. Values are means, and error bars indicate standard deviation. BMD, bone mineral density; P1NP, procollagen type I amino-terminal propeptide a

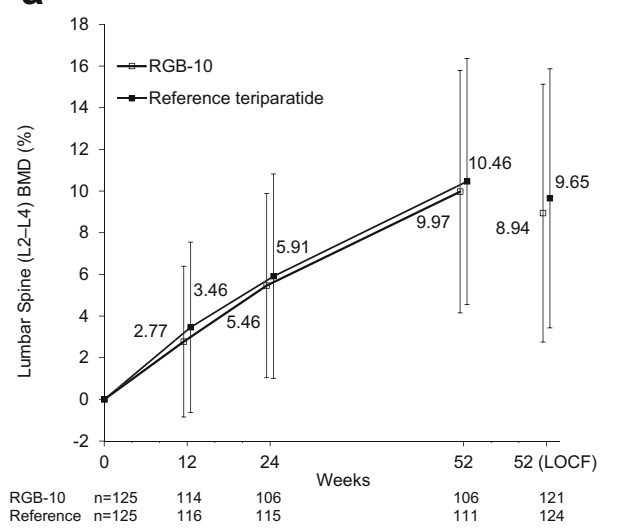

C

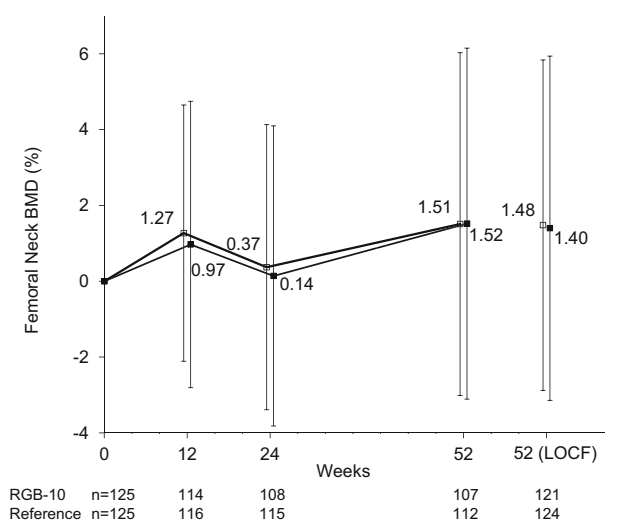

b

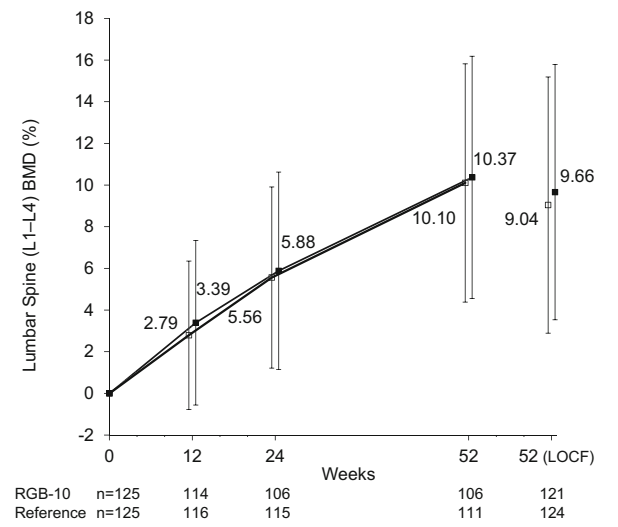

d

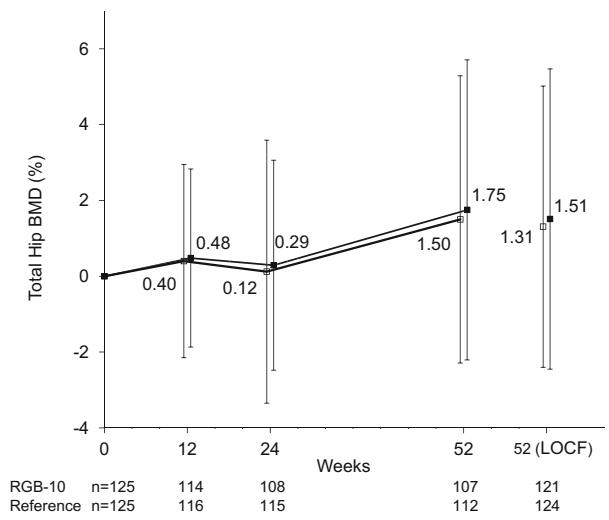

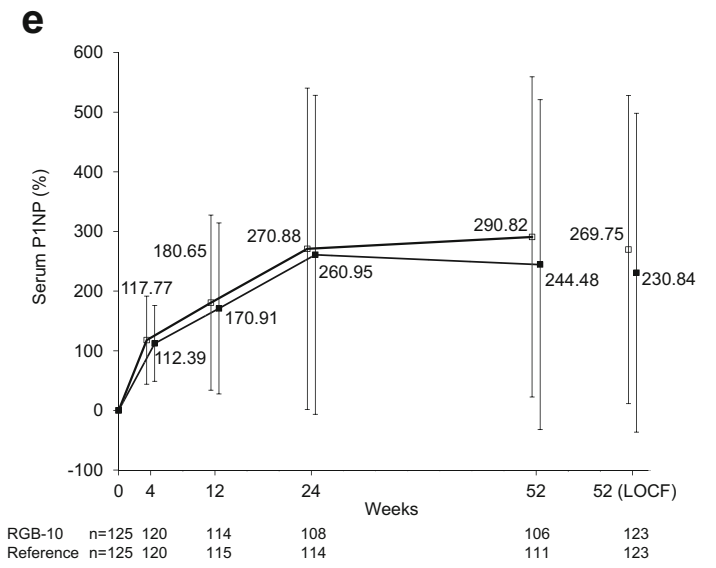

reference teriparatide group (Table 3). In the reference teriparatide group, one patient was additionally already positive for anti-teriparatide antibodies at baseline and continued to show positivity after administration of the investigational drug. In both patients positive for antiteriparatide antibodies, no neutralizing activity was detected, no AEs with a potential relationship to antiteriparatide antibody production (such as hypoparathyroidism, hypocalcemia, hyperphosphatemia, anaphylactic shock, or drug eruption) were reported, and no decrease in efficacy was observed.

\section{Discussion}

This phase 3 study evaluated the similarity in efficacy and safety of RGB-10 and reference teriparatide administered to patients with osteoporosis at high risk of fracture for 52 weeks.

Although biosimilars to teriparatide, including RGB-10, have previously been reported in highly regulated regions $[15,19]$, to the best of our knowledge, this is the first phase 3 study of a biosimilar teriparatide with the primary objective of demonstrating therapeutic equivalence in terms of BMD assessed at 12 months. RGB-10 is the first biosimilar 
Table 3 Adverse events and immunogenicity

\begin{tabular}{|c|c|c|c|c|}
\hline \multirow[t]{2}{*}{ Events } & \multicolumn{2}{|c|}{ RGB-10 $(n=125)$} & \multicolumn{2}{|c|}{ Reference teriparatide $(n=125)$} \\
\hline & Patients & Incidence $(\%)$ & Patients & Incidence $(\%)$ \\
\hline All AEs & 107 & 85.6 & 107 & 85.6 \\
\hline All AEs related to investigational drug & 59 & 47.2 & 57 & 45.6 \\
\hline SAEs & 3 & 2.4 & 6 & 4.8 \\
\hline Deaths & 0 & 0.0 & 0 & 0.0 \\
\hline AEs leading to study discontinuation & 15 & 12.0 & 11 & 8.8 \\
\hline \multicolumn{5}{|c|}{ AEs reported in $>5 \%$ patients in either group } \\
\hline Nasopharyngitis & 32 & 25.6 & 36 & 28.8 \\
\hline Injection site erythema & 13 & 10.4 & 9 & 7.2 \\
\hline Nausea & 13 & 10.4 & 9 & 7.2 \\
\hline Injection site bruising & 12 & 9.6 & 4 & 3.2 \\
\hline Blood uric acid increased & 9 & 7.2 & 12 & 9.6 \\
\hline Headache & 9 & 7.2 & 11 & 8.8 \\
\hline Constipation & 8 & 6.4 & 10 & 8.0 \\
\hline Contusion & 8 & 6.4 & 7 & 5.6 \\
\hline Arthralgia & 8 & 6.4 & 6 & 4.8 \\
\hline Abdominal discomfort & 8 & 6.4 & 5 & 4.0 \\
\hline Blood alkaline phosphatase increased & 7 & 5.6 & 9 & 7.2 \\
\hline Back pain & 7 & 5.6 & 5 & 4.0 \\
\hline \multicolumn{5}{|l|}{ Immunogenicity } \\
\hline Anti-teriparatide antibodies ${ }^{a}$ & 0 & 0.0 & 1 & 0.8 \\
\hline Neutralizing activities $^{\mathrm{a}}$ & 0 & 0.0 & 0 & 0.0 \\
\hline Anti-teriparatide antibodies ${ }^{b}$ & 0 & 0.0 & 2 & 1.6 \\
\hline Neutralizing activities ${ }^{\mathrm{b}}$ & 0 & 0.0 & 0 & 0.0 \\
\hline
\end{tabular}

AEs reported in the treatment period and follow-up period were collected

${ }^{a}$ Patients newly positive for anti-teriparatide antibodies after the first administration of the investigational drug

${ }^{\mathrm{b}}$ Patients positive for anti-teriparatide antibodies at least once in the study

$A E$ adverse event, $S A E$ serious adverse event

teriparatide to have been granted marketing authorization in the EU, and the first biosimilar to have been approved along the principles of the specifically tailored approach. In line with pertinent EMA guidelines, given the simple molecular structure and the well-established mechanism of action of teriparatide as well as the robust quality and non-clinical comparability data for RGB-10 and the European reference product, a comparative PK/PD study in healthy volunteers was considered sufficient for granting marketing authorization. A phase 3 study of another biosimilar teriparatide conducted in the USA evaluated the immunogenicity of the biosimilar and the reference formulations of teriparatide as its primary objective.

In the present study, equivalence in efficacy between RGB10 and reference teriparatide was established: the betweengroup difference of the primary efficacy endpoint of percent change from baseline to 52 weeks in lumbar spine (L2-L4) BMD was $-0.65 \%$, and its two-sided $95 \%$ CI $(-2.17 \%$ to $0.87 \%$ ) was within the pre-specified equivalence margin of $\pm 2.8 \%$. Furthermore, the percent change from baseline to 52 weeks in lumbar spine (L2-L4) (RGB-10, 8.94\%; reference teriparatide, $9.65 \%$ ) was similar to that reported in a phase 3 study of reference teriparatide in Japan (reference teriparatide, 9.82\%) that we used as a reference in planning the design of this study and that had a similar patient population to that of our study [7].

In patients who completed 52 weeks of treatment with RGB-10 or teriparatide, the percent changes in lumbar spine (L2-L4) BMD from baseline to 52 weeks were $9.97 \pm 5.82 \%$ and $10.46 \pm 5.90 \%$, respectively, which were similar to that in the reference phase 3 study $(10.04 \pm 5.23 \%)$ [7]. These changes in patients who completed the study were numerically larger than the results of the primary efficacy endpoint, where missing data were imputed with LOCF, suggesting that completion of 52 weeks of treatment was an important determining factor of the effect of teriparatide on BMD. Considering the low long-term adherence and persistence of teriparatide treatment, a general feature of osteoporosis medications [20], 
these results support the previously reported importance of intervention aimed at maintaining patient adherence and persistence to teriparatide [21-24].

Lumbar spine BMD was measured primarily from L2 to L4 in the present study, as in the reference phase 3 study [7]. The trend in lumbar spine (L1-L4) BMD change in this study was similar to that of lumbar spine (L2-L4). In addition, the twosided $95 \%$ CI of between-group difference in the percent change to 52 weeks in lumbar spine (L1-L4) BMD ($2.05 \%$ to $0.93 \%$ ) was also within $\pm 2.8 \%$. These results strongly support the equivalence in efficacy between the two groups. The results for other efficacy endpoints (BMD change in femoral neck and total hip, serum P1NP change, and fracture incidence) were also similar between the two groups, which further supported the comparable efficacy of RGB-10 to reference teriparatide.

The safety profiles of RGB-10 and reference teriparatide were comparable. No clinically significant differences were observed between RGB-10 and reference teriparatide in terms of the type, incidence, severity, and seriousness of AEs. The incidence of AEs leading to study discontinuation was numerically higher in the RGB-10 group than in the reference teriparatide group. However, this higher incidence was considered to be of no clinical significance because the majority of AEs leading to study discontinuation in the RGB-10 group were events observed with the use of reference teriparatide in clinical trials and postmarketing exposure (Online Resource 3) [1]; there was no high incidence of any AEs leading to study discontinuation in the RGB-10 group; and most of the AEs leading to study discontinuation were mild to moderate in severity. The numerically higher incidence of injection site bruising in the RGB-10 group was considered to be an accidental finding because the severity, outcome, and onset time of injection site bruising were similar between the groups; the formulation of RGB-10 is the same as that of reference teriparatide; and the irritant potential of both drugs was considered to be similar based on findings in a repeat-dose toxicity study in rats (data on file). The difference in injection device was unlikely to have contributed to the higher incidence of injection site bruising in the RGB-10 group because both devices were comparable in terms of injection procedure and dose accuracy, and the same type of needle was used for both groups.

Regarding immunogenicity, the incidence of antiteriparatide antibodies was comparably low, with antibodies detected in no patients in the RGB-10 group and in two patients in the reference teriparatide group, none of which tested positive for neutralizing activity. This finding was consistent with that of a previously reported study on reference teriparatide, where $2.8 \%$ of the patients receiving $20 \mu \mathrm{g}$ daily teriparatide were positive for anti-teriparatide antibodies [1, 6], although the duration of the treatment in our study slightly differed from that described in the previous report (up to 52 weeks vs 18 months [mean]). Consequently, the immunogenic potential of RGB-10 was considered not to exceed that of the reference teriparatide

Although this study was conducted in a rater-blinded manner, patients were also largely unaware of their own assigned treatment group. To minimize potential bias, the name of the injection device was masked, and although patients knew that the injection devices for the two investigational drugs differed in appearance, they were not informed of their treatment group. Moreover, the blinding status of patients was considered unlikely to affect the efficacy data because BMD is an objective value, and raters, including the central assessment facility, remained blinded. This was also applicable to the safety data as the investigators involved in safety assessments were blinded. We therefore consider that any potential bias caused by the unblinding of patients was sufficiently minimized so as not to have substantially affected the efficacy and safety data in the present study.

A strength of the present study is that the patient population was similar to that found in clinical settings in Japan as the inclusion criteria were determined according to clinical guidelines, including Japanese guidelines for the prevention and treatment of osteoporosis [18].

The present study had some limitations. First, because the patients participating in the study were all of Asian ethnicity, the effects of RGB-10 in patients of other ethnic origins were unknown. However, because teriparatide meets most of the criteria for being less likely to be sensitive to intrinsic and extrinsic ethnic factors, similar efficacy and safety are expected in other populations [25]. Second, because this study was not designed to compare the effect of teriparatide on the reduction of fractures between the groups, the limited duration and the number of subjects did not allow for statistical assessment of the incidence of fractures. However, given that BMD is a widely accepted surrogate marker for fractures, accounting for up to a $30-41 \%$ reduction in the risk of vertebral fractures in the case of teriparatide treatment, and in view of the highly comparable changes in BMD in the RGB-10 and reference teriparatide groups observed in our study, the two compounds are expected to be therapeutically equivalent in terms of their effect on reducing the risk of fragility fractures [26].

In conclusion, the results of our study established therapeutic equivalence and comparable safety, including immunogenicity, between RGB-10 and the reference teriparatide, further substantiating the claim of biosimilarity.

Acknowledgments The authors wish to acknowledge the patients who participated in the clinical trial and the investigators and staff at the study sites. The authors also acknowledge Ryosuke Murai of Mochida Pharmaceutical Co., Ltd. for medical writing assistance, and thank Clare Cox, PhD, from Liwen Bianji, Edanz Editing China (www.liwenbianji.cn/ac), for editing the English text of a draft of this manuscript. 
Funding This study was funded by Mochida Pharmaceutical Co., Ltd.

\section{Compliance with ethical standards}

Conflicts of interest $\mathrm{HH}$ has received consulting fees for this study from Mochida Pharmaceutical and lecture fees or grants outside the submitted work from Asahi Kasei Pharma Corp., Astellas Pharma Inc., Chugai Pharmaceutical Co., Ltd., Eisai Co., Ltd., Eli Lilly Japan Co., Ltd., Mitsubishi Tanabe Pharma Corp., Ono Pharmaceutical Co., Ltd., Pfizer Inc., Taisho Toyama Pharmaceutical Co., Ltd., Takeda Pharmaceutical Co., Ltd., Teijin Pharma Co., Ltd. and Daiichi Sankyo Co., Ltd. RN, YY, MW, and MT are employees of Mochida Pharmaceutical. All authors declared no other relationships/conditions/circumstances that present a potential conflict of interest, in accordance with the "Transparency guideline for the Relation between Corporate Activities and Medical Institutions" prepared by the Japan Pharmaceutical Manufacturers Association, corresponding in Japan to the Physicians Payment Sunshine Act.

Ethical approval All procedures performed in studies involving human participants were in accordance with the ethical standards of the institutional and/or national research committee and with the 1964 Helsinki declaration and its later amendments or comparable ethical standards.

Informed consent Informed consent was obtained from all individual participants included in the study.

Open Access This article is distributed under the terms of the Creative Commons Attribution-NonCommercial 4.0 International License (http:// creativecommons.org/licenses/by-nc/4.0/), which permits any noncommercial use, distribution, and reproduction in any medium, provided you give appropriate credit to the original author(s) and the source, provide a link to the Creative Commons license, and indicate if changes were made.

\section{References}

1. European Medicines Agency (2018) Forsteo: EPAR - product information (SmPC). https://www.ema.europa.eu/documents/ product-information/forsteo-epar-product-information_en.pdf. Accessed 27 December 2018

2. Eli Lilly and Company (2013) FORTEO (teriparatide [rDNA origin] injection): Label. https://www.accessdata.fda.gov/drugsatfda docs/label/2013/021318s036lbl.pdf. Accessed 27 December 2018

3. Blick SK, Dhillon S, Keam SJ (2008) Teriparatide: a review of its use in osteoporosis. Drugs 68(18):2709-2737

4. Fu Q, Jilka RL, Manolagas SC, O'Brien CA (2002) Parathyroid hormone stimulates receptor activator of NFKB ligand and inhibits osteoprotegerin expression via protein kinase a activation of cAMP-response element-binding protein. J Biol Chem 277(50): 48868-48875

5. Dobnig H, Turner RT (1997) The effects of programmed administration of human parathyroid hormone fragment (1-34) on bone histomorphometry and serum chemistry in rats. Endocrinology 138(11):4607-4612

6. Neer RM, Arnaud CD, Zanchetta JR, Prince R, Gaich GA, Reginster JY, Hodsman AB, Eriksen EF, Ish-Shalom S, Genant HK, Wang O, Mitlak BH (2001) Effect of parathyroid hormone
(1-34) on fractures and bone mineral density in postmenopausal women with osteoporosis. N Engl J Med 344(19):1434-1441

7. Miyauchi A, Matsumoto T, Sugimoto T, Tsujimoto M, Warner MR, Nakamura T (2010) Effects of teriparatide on bone mineral density and bone turnover markers in Japanese subjects with osteoporosis at high risk of fracture in a 24-month clinical study: 12-month, randomized, placebo-controlled, double-blind and 12-month open-label phases. Bone 47(3):493-502

8. McClung MR, San Martin J, Miller PD, Civitelli R, Bandeira F, Omizo M, Donley DW, Dalsky GP, Eriksen EF (2005) Opposite bone remodeling effects of teriparatide and alendronate in increasing bone mass. Arch Intern Med 165(15):1762-1768

9. Orwoll ES, Scheele WH, Paul S, Adami S, Syversen U, Diez-Perez A, Kaufman JM, Clancy AD, Gaich GA (2003) The effect of teriparatide [human parathyroid hormone (1-34)] therapy on bone density in men with osteoporosis. J Bone Miner Res 18(1):9-17

10. European Medicines Agency and the European Commission (2017) Biosimilars in the EU information guide for healthcare professionals. https:/www.ema.europa.eu/documents/leaflet/biosimilarseu-information-guide-healthcare-professionals_en.pdf. Accessed 27 December 2018

11. Medicines for Europe (2016) Biosimilar medicines handbook. 3rd edn. https://www.medicinesforeurope.com/wp-content/uploads/ 2016/04/Medicines-for- Europe_BIOSIMILARS_INT_web.pdf\# search $=\% 27$ Biosimilar + medicines + handbook $\% 27$. Accessed 22 Nov 2018

12. European Medicines Agency (2014) Guideline on similar biological medicinal products. CHMP/437/04 Rev 1. https://www.ema. europa.eu/documents/scientific-guideline/guideline-similarbiological-medicinal-products-rev1_en.pdf. Accessed 27 December 2018

13. US Food and Drug Administration (2015) scientific considerations in demonstrating biosimilarity to a reference product. https://www. $\mathrm{f} \mathrm{d} \mathrm{a.g} \mathrm{o} \mathrm{v} \mathrm{/} \mathrm{d} \mathrm{o} \mathrm{w} \mathrm{n} \mathrm{l} \mathrm{o} \mathrm{a} \mathrm{d} \mathrm{s} \mathrm{/} \mathrm{D} \mathrm{r} \mathrm{u} \mathrm{g} \mathrm{s} \mathrm{/}$ GuidanceComplianceRegulatoryInformation/Guidances/ UCM291128.pdf. Accessed 27 December 2018

14. Ministry of Health, Labour and Welfare, Japan (2009) Guideline for the quality, safety, and efficacy Assurance of Follow-on Biologics. PFSB/ELD Notification No. 0304007. http://www.pmda.go.jp/ files/000153851.pdf. Accessed 27 December 2018

15. Takács I, Jókai E, Kováts DE, Aradi I (2018) The first biosimilar approved for the treatment of osteoporosis: results of a comparative pharmacokinetic/pharmacodynamic study. Osteoporos Int 30:675683. https://doi.org/10.1007/s00198-018-4741-0

16. European Medicines Agency (2016) Terrosa: EPAR. EMA/ 833583/2016; EMEA/H/C/003916. https://www.ema.europa.eu/ documents/overview/terrosa-epar-summary-public_en.pdf. Accessed 27 December 2018

17. Orimo H, Hayashi Y, Fukunaga M, Sone T, Fujiwara S, Shiraki M, Kushida K, Miyamoto S, Soen S, Nishimura J, Oh-Hashi Y, Hosoi T, Gorai I, Tanaka H, Igai T, Kishimoto H, Osteoporosis Diagnostic Criteria Review Committee: Japanese Society for Bone and Mineral Research (2001) Diagnostic criteria for primary osteoporosis: year 2000 revision. J Bone Miner Metab 19(6):331-337

18. Orimo H (2015) Japanese 2015 guidelines for prevention and treatment of osteoporosis. Life Science Publishing, Tokyo

19. ClinicalTrials.gov (2018) A comparison of PF708 and Forteo in osteoporosis patients. https://clinicaltrials.gov/ct2/show/ NCT03002428. Accessed 27 December 2018

20. Yu S, Burge RT, Foster SA, Gelwicks S, Meadows ES (2012) The impact of teriparatide adherence and persistence on fracture outcomes. Osteoporos Int 23(3):1103-1113 
21. Nogues X, Luz Rentero M, Rodriguez AL (2014) Use of an educational support program to assist patients receiving injectable osteoporosis treatment: experience with teriparatide. Curr Med Res Opin 30(2):287-296

22. Briot K, Ravaud P, Dargent-Molina P, Zylberman M, Liu-Leage S, Roux C (2009) Persistence with teriparatide in postmenopausal osteoporosis; impact of a patient education and follow-up program: the French experience. Osteoporos Int 20(4):625-630

23. Tamone C, Fonte G, Panico A, Molinatti PA, D'Amelio P, Isaia GC (2012) Impact of a phone follow-up program on persistence with teriparatide or PTH(1-84) treatment. Calcif Tissue Int 90(4):272-278

24. Sato M, Tsujimoto M, Kajimoto K, Uetake H, Shimoda H, Fujiwara S (2018) Effect of a patient-support program on once-daily teriparatide adherence and persistence in the Japan fracture observational study (JFOS). Arch Osteoporos 13(1):74. https://doi.org/ 10.1007/s11657-018-0487-8
25. Tsujimoto M, Uenaka K, Iwata A, Higashiuchi Y, Sowa H (2012) Effects of teriparatide in Japanese and non-Japanese populations: bridging findings on pharmacokinetics and efficacy. J Bone Miner Metab 30(3):326-337

26. Chen P, Miller PD, Delmas PD, Misurski DA, Krege JH (2006) Change in lumbar spine BMD and vertebral fracture risk reduction in teriparatide-treated postmenopausal women with osteoporosis. J Bone Miner Res 21(11):1785-1790

Publisher's note Springer Nature remains neutral with regard to jurisdictional claims in published maps and institutional affiliations. 